ORIGINAL ARTICLE

\title{
Exome sequencing of Pakistani consanguineous families identifies 30 novel candidate genes for recessive intellectual disability
}

\author{
S Riazuddin ${ }^{1,2,16}$, M Hussain 1,3,4,5,16, A Razzaq ${ }^{3,4,5,16}$, Z lqbal ${ }^{3,14,16}$, M Shahzad $^{1,2}$, DL Polla ${ }^{3,6}$, Y Song $^{7}$, E van Beusekom ${ }^{3}$, AA Khan $^{5}$, \\ L Tomas-Roca $^{3}$, M Rashid ${ }^{3,4,5}$, MY Zahoor ${ }^{5}$, WM Wissink-Lindhout ${ }^{3}$, MAR Basra ${ }^{5}$, M Ansar ${ }^{3,5,15}$, Z Agha $^{3,8}, \mathrm{~K}$ van Heeswijk $^{3}$, F Rasheed R $^{5}$, \\ M Van de Vorst ${ }^{3}$, JA Veltman ${ }^{3,9}$, C Gilissen $^{3}$, J Akram², T Kleefstra ${ }^{3}$, MZ Assir ${ }^{4}$, UK10K ${ }^{10}$, D Grozeva ${ }^{11}$, K Carss ${ }^{12}$, FL Raymond ${ }^{11}$, \\ TD O'Connor ${ }^{7}$, SA Riazuddin ${ }^{13}$, SN Khan ${ }^{5}$, ZM Ahmed ${ }^{1}$, APM de Brouwer ${ }^{3}$, H van Bokhoven $^{3,17}$ and S Riazuddin ${ }^{2,4,17}$
}

Intellectual disability (ID) is a clinically and genetically heterogeneous disorder, affecting 1-3\% of the general population. Although research into the genetic causes of ID has recently gained momentum, identification of pathogenic mutations that cause autosomal recessive ID (ARID) has lagged behind, predominantly due to non-availability of sizeable families. Here we present the results of exome sequencing in 121 large consanguineous Pakistani ID families. In 60 families, we identified homozygous or compound heterozygous DNA variants in a single gene, 30 affecting reported ID genes and 30 affecting novel candidate ID genes. Potential pathogenicity of these alleles was supported by co-segregation with the phenotype, low frequency in control populations and the application of stringent bioinformatics analyses. In another eight families segregation of multiple pathogenic variants was observed, affecting 19 genes that were either known or are novel candidates for ID. Transcriptome profiles of normal human brain tissues showed that the novel candidate ID genes formed a network significantly enriched for transcriptional co-expression $(P<0.0001)$ in the frontal cortex during fetal development and in the temporal-parietal and sub-cortex during infancy through adulthood. In addition, proteins encoded by 12 novel ID genes directly interact with previously reported ID proteins in six known pathways essential for cognitive function $(P<0.0001)$. These results suggest that disruptions of temporal parietal and sub-cortical neurogenesis during infancy are critical to the pathophysiology of ID. These findings further expand the existing repertoire of genes involved in ARID, and provide new insights into the molecular mechanisms and the transcriptome map of ID.

Molecular Psychiatry (2017) 22, 1604-1614; doi:10.1038/mp.2016.109; published online 26 July 2016

\section{INTRODUCTION}

Intellectual disability (ID) is a common neurodevelopmental disorder with an onset of cognitive impairment before the age of 18 years ${ }^{1-3}$ and is characterized by significant limitations in intellectual functioning and adaptive behavior. ${ }^{1}$ The disease affects $1-3 \%$ of the world population; however, its prevalence in the developing world is almost twice that of the affluent world. ${ }^{4,5}$ The causes of nearly $40 \%$ of ID remain ambiguous. ${ }^{6}$ Among the known causes, $~ 50 \%$ of ID cases have an environmental etiology such as poor nutrition, multiple pregnancies with little gap, prenatal/perinatal brain ischemia, postnatal infections and inadequate medical services.
The other half of ID cases has a genetic etiology, such as chromosomal abnormalities or mutations in specific genes. ${ }^{3,5}$

In the western world, de novo heterozygous mutations and genomic copy number changes account for the majority of ID cases. ${ }^{7}$ In contrast, recessive ID appears to be more common in consanguineous populations. Identification of gene mutations that cause non-syndromic autosomal recessive ID (ARID) has been notoriously slow because of the scarcity of sizeable families. By 2006, only three genes, CC2D1A, CRBN and PRSS12 had been associated with ARID. $^{8-10}$ After 2006, research studies involving highly inbred populations from North Africa, the Middle East and South East Asia,

${ }^{1}$ Department of Otorhinolaryngology_-Head and Neck Surgery, University of Maryland, School of Medicine, Baltimore, MD, USA; ${ }^{2}$ Center for Genetic Diseases, Shaheed Zulfiqar Ali Bhutto Medical University, Pakistan Institute of Medical Sciences, Islamabad, Pakistan; ${ }^{3}$ Department of Human Genetics, Donders Institute for Brain, Cognition and Behaviour, Radboud University Medical Center, Nijmegen, The Netherlands; ${ }^{4}$ Allama Iqbal Medical College, University of Health Sciences, Lahore, Pakistan; ${ }^{5}$ National Centre of Excellence in Molecular Biology, University of The Punjab, Lahore, Pakistan; ${ }^{6}$ Center for Genetic Diseases, CAPES Foundation, Ministry of Education of Brazil, Brasília, Brazil; ${ }^{7}$ Institute for Genome Sciences and Program in Personalized and Genomic Medicine, University of Maryland, School of Medicine, Baltimore, MD, USA; ${ }^{8}$ Department of Biosciences, Faculty of Science, COMSATS Institute of Information Technology, Islamabad, Pakistan; ${ }^{9}$ Department of Clinical Genetics, GROW School for Oncology and Developmental Biology, Maastricht University Medical Centre, Maastricht, The Netherlands; ${ }^{10}$ The Wellcome Trust Sanger Institute, Wellcome Trust Genome Campus, Cambridge, UK; ${ }^{11}$ Department of Medical Genetics, Cambridge Institute for Medical Research, University of Cambridge, Cambridge, UK; ${ }^{12}$ Department of Haematology, University of Cambridge, Cambridge, UK and ${ }^{13}$ Department of Ophthalmology, The Wilmer Eye Institute, Johns Hopkins University School of Medicine, Baltimore, MD, USA. Correspondence: Professor S Riazuddin, Department of Otorhinolaryngology-Head and Neck Surgery, University of Maryland School of Medicine, Baltimore, MD 21201, USA or Professor H van Bokhoven, Department of Human Genetics, Donders Institute for Brain, Cognition and Behaviour, Radboud University Medical Center, PO BOX 9101, Nijmegen 6500 HB, The Netherlands or Professor S Riazuddin, Allama labal Medical College, University of Health Sciences, Lahore 54550, Pakistan.

E-mail: sriazuddin@smail.umaryland.edu or Hans.vanbokhoven@radboudumc.nl or riazuddin@aimrc.org

${ }^{14}$ Current address: Department of Neurology, Oslo University Hospital, Oslo, Norway.

${ }^{15}$ Current address: Department of Genetic Medicine and Development, University of Geneva, Geneva, Switzerland.

${ }^{16}$ These authors share equal first authorship.

${ }^{17}$ These authors share equal senior authorship.

Received 16 November 2015; revised 18 May 2016; accepted 1 June 2016; published online 26 July 2016 
greatly accelerated the pace of identification of gene mutations that cause ARID. These studies were further augmented by the use of next-generation sequencing. In 2011, Najmabadi et al. ${ }^{11}$ applied targeted next-generation sequencing to 136 consanguineous Iranian families, in which homozygosity mapping had previously identified a locus, and reported 23 known and 50 new candidate ARID genes. More recently, exome sequencing (ES) of 143 large consanguineous Saudi Arabian families revealed 33 novel candidate genes involved in different neurological disorders. ${ }^{12}$ These studies further highlighted the clinical and genetic complexity of ID and other neurodevelopmental disorders. Intriguingly, not a single gene with pathogenic variants emerged across various populations. Despite this progress, a large number of potential pathogenic gene mutations remain unidentified and warrant further studies in extended families from communities with endogamy.

Consanguineous marriages are practiced by about $20 \%$ of the human population, and the extent of consanguinity varies among different citizenry in the world. The rate of consanguineous marriages is $38 \%$ in Iran, ${ }^{13,14}$ over $40 \%$ in several Middle Eastern countries $^{15}$ and above $50 \%$ in Pakistan. ${ }^{16}$ The elevated level of endogamy in Pakistan has led to the increased prevalence of genetic disorders, including ARID, with an average of 1.1 cases of severe ID and 6.2 cases of mild ID per 100 live births. ${ }^{17}$ The present study is designed to identify pathogenic gene mutations that cause ARID in the highly inbred population of Pakistan. In all, 121 families of 8 different ethnicities, exhibiting recessive ID, were enrolled mostly from the rural population of Pakistan. Through a systematic exome-sequencing approach we have identified potential pathogenic variants in 68 of these families: 30 families with a single homozygous DNA variant affecting previously known ID genes and another 30 families with a single homozygous or compound heterozygous variants in novel candidate ID genes. In eight families multiple homozygous variants were identified. We report the phenotype-genotype relationships, the predicted pathogenicity of the newly discovered candidate genes, their co-expression in functional networks in the developing and adult human brain, and possible involvement in various cellular processes.

\section{MATERIALS AND METHODS}

Family ascertainment and phenotype analysis

This study was approved by the Institutional Review Board of the Centre of Excellence in Molecular Biology (CEMB), University of the Punjab, Lahore, Pakistan and the Medical Ethical Committee Arnhem-Nijmegen, The Netherlands. The families of our cohort were obtained mainly from the rural populations of all five provinces of Pakistan (Supplementary Tables S1 and S2). Written informed consents were obtained from healthy adult subjects and the parents/legal guardians of minor subjects and ID patients. Specific informed consent was obtained for showing images of patients (Supplementary Figure S1). Participating individuals were evaluated with medical history interviews, and ID-related phenotypic features of patients were recorded. All affected individuals were clinically evaluated by both a geneticist and a general medical practitioner, with particular attention to neurological, morphological, ophthalmological, dermatological and skeletal symptoms. Photographs of the face and uncovered limbs were also taken (Supplementary Figure S1). In some cases, magnetic resonance imaging and computerized tomography scans were also obtained. A description of developmental milestones was used to evaluate the severity of ID. ${ }^{18}$ Only families with confirmed existence of cognitive dysfunction in the affected members were included in this study. Peripheral blood samples were collected from participating subjects. Genomic DNA was isolated following standard procedures. ${ }^{19}$

\section{Exome sequencing and variant selection}

Exome enrichment and high-throughput sequencing were performed at the Radboudumc (Nijmegen, The Netherlands), The Wellcome Trust Sanger Institute (Hinxton, as part of the UK10K study) and the University of Maryland as previously described. ${ }^{20}$ Selection of high-quality, potentially pathogenic variants was performed using seven major filtration steps (Supplementary Figure S2 and Supplementary Methods).

\section{Sanger sequencing}

ES results were confirmed by Sanger sequencing. Primers for the amplification of the exons carrying variants were designed by using Primer3 (ref. 21; Supplementary Tables S9 and S10).

Predicted involvement of candidate genes in cellular processes and pathways

We researched the available literature and extracted all reported proteinprotein interactions involving the known and the newly identified ID proteins and then entered them into a set of protein-protein interaction networks via the STRING ${ }^{22}$ and MATISSE ${ }^{23}$ algorithms, which are included in the EXPANDER package tools. ${ }^{24}$

\section{Expression of candidate genes in the human brain}

Brain expression analyses for new ARID genes were conducted using existing data sets in the BrainSpan: Atlas of the Developing Human Brain. ${ }^{25}$ Normalized gene expression levels for 26 different brain tissues from 31 different developmental periods were obtained from the BrainSpan RNAseq data set v3 (http://brainspan.org) of the developing human brain.

\section{Co-expression network analysis}

Networks of functionally co-expressed genes were identified by using the model of Gulsuner et al. ${ }^{26}$ Using the BrainSpan database, gene pairs are defined as connected if the absolute value of the Pearson's correlation coefficient is $>0.8$ for their expression levels in different brain regions (frontal cortex, temporal-parietal, sensory-motor and sub-cortical) and different developmental stages (fetal, infancy to late childhood and adolescence to adulthood), totaling 12 different networks. For each of these networks the numbers of edges (connections) between each of the novel ID genes was calculated. Random simulations of 10000 replicates of an equal number of genes randomly selected from the BrainScan database were conducted to establish the significance of the connections. This provided a distribution of edges to estimate an empirical $P$-value for our novel ID genes.

In addition, we also randomly selected genes from the BrainScan database with an HGNC gene symbol, as well as at least one connection in the temporal region network. We compared an equal number of genes in this set with our ID genes that passed the same filtering criterion, which provided more conservative and consistent $P$-values.

\section{RESULTS}

Recruitment of families

We enrolled a cohort of 121 ID families with a likely autosomal recessive inheritance pattern mostly from the rural areas of Punjab, Sindh, Baluchistan, Khyber Pakhtoon Khawa and Northern areas. The enrolled families comprise eight ethnic groups, namely Punjabi (68.6\%), Siraiki (10.8\%), Pathan (9\%), Urdu speaking (7.8\%) and others (Sindhi, Afghan, Baloch and Kashmiri (3.8\%); Supplementary Table S1). Every family had two or more affected individuals (except PKMR51a) and 111 families had consanguineous unions, while in 74 families the affected individuals were present in separate sibships (Supplementary Table S2).

\section{Genetic analysis}

Before ES, the presence of disease-causing copy number variations was excluded by SNP microarray analysis (Affymetrix $250 \mathrm{~K}$ SNP array or higher coverage). ES was carried out for DNA samples of 1-3 individuals per family. Selection of the nucleotide variants was performed using the seven-tier filtering strategy (Supplementary Figure S2). Sanger sequencing confirmed 359 candidate pathogenic variants. Of these, 80 variants in 77 genes segregated with the ID phenotype. There are six categories of evidence that we are using in this study to support an assertion that a variant is potentially pathogenic. These are (1) segregation analysis; (2) either absence in the dbSNP142 database or a very low allele frequency $(<0.01)$ in the ExAC Browser database; (3) absence in 213 ethnically matched control individuals; (4) a CADD score $>20$; 
Table 1. Variants identified in known genes for intellectual disability or related disorders

\begin{tabular}{|c|c|c|c|c|c|c|c|c|c|c|}
\hline Family & Gene & Position $^{\mathrm{a}}$ & Transcript & cDNA mutation ${ }^{\mathrm{b}}$ & Protein change & Variation & $\begin{array}{c}\text { CADD score } \\
\text { v1.3 }\end{array}$ & $\begin{array}{l}\text { ExAC allele frequency } \\
\qquad \times 10^{-6}\end{array}$ & OMIM & Phenotype $^{c}$ \\
\hline PKMR08 & GNE & Chr9:36217445C $>T$ & NM_005476.5 & c. $2086 \mathrm{G}>\mathrm{A}$ & p.(Val696Met) & Missense & 32.0 & 1942 & 603824 & D \\
\hline PKMR29 & РОMT2 & Chr14:77765840T $>C$ & NM_013382.5 & c. $881 \mathrm{~A}>\mathrm{G}$ & p.(Tyr294Cys) & Missense & 27.9 & 0 & 607439 & $\mathrm{O}$ \\
\hline PKMR36 & APTX & Chr9:32984803delC & NM_001195248.1 & c.638delG & p.(Arg213Leufs*15) & Frameshift & NA & 8.238 & 606350 & $\mathrm{O}$ \\
\hline PKMR42 & VPS13B & Chr8:100654333C >T & NM_017890.4 & c. $5590 \mathrm{C}>\mathrm{T}$ & p. $\left(\mathrm{G} \ln 1864^{*}\right)$ & Nonsense & NA & 0 & 607817 & $\mathrm{O}$ \\
\hline PKMR51a & TSHR & Chr14:81610687C $>T$ & NM_000369.2 & c. $2285 C>T$ & p.(Thr762Met) & Missense & 25.3 & 8.247 & 603372 & D \\
\hline PKMR61a & SCN1A & Chr2:166848284G $>$ A & NM 001165963.1 & $c .5501 C>T$ & p.(Ala1834Val) & Missense & 32 & 8.241 & $182389^{d}$ & 0 \\
\hline PKMR79 & AP4M1 & Chr7:99704430delG & NM_004722.3 & c.1287delG & p.(Arg429Serfs*15) & Frameshift & NA & 0 & 602296 & $\mathrm{O}$ \\
\hline PKMR82 & KCNA2 & Chr1:111147212G > A & NM_004974.3 & c. $193 C>T$ & p. $($ Arg65*) & Nonsense & NA & 8.239 & $176262^{d}$ & $\begin{array}{c}\text { C, inheritance } \\
\text { D }\end{array}$ \\
\hline PKMR85 & MED23 & Chr6:131941859T $>C$ & NM_004830.3 & c.506A $>$ G & p.(Tyr169Cys) & Missense & 24.6 & 0 & 605042 & C \\
\hline PKMR86 & FRAS1 & Chr4:79202579C $>T$ & NM_025074.6 & c. $1099 \mathrm{C}>\mathrm{T}$ & p.(Arg367Cys) & Missense & 24.4 & 43.14 & 607830 & $\mathrm{O}$ \\
\hline PKMR87 & MAN2B1 & Chr19:12763176C > G & NM_000528.3 & c. $1928+1 C>G$ & p.(Phe642Phefs*2) & $\begin{array}{l}\text { Aberrant } \\
\text { splicing }\end{array}$ & NA & 0 & 609458 & $\mathrm{O}$ \\
\hline PKMR97 & MFSD2A & Chr1:40431565C $>T$ & NM_001136493.2 & $c .632 \mathrm{C}>\mathrm{T}$ & p.(Thr211Met) & Missense & 33 & 8.239 & 614397 & C \\
\hline PKMR99 & SYNE1 & Chr6:152819877C> G & NM_182961.3 & c.939G $>C$ & p.(Lys313Asn) & Missense & 21.9 & 28.33 & 608441 & D \\
\hline PKMR102 & $A S P M$ & Chr1:197087007C $>T$ & NM_018136.4 & c.3977G $>$ A & p. (Trp1326*) & Nonsense & NA & 8.255 & 605481 & C \\
\hline PKMR105 & ZNF41 & ChrX:47315776C >T & NM_007130.2 & c.94G $>$ A & p.(Val32Met) & Missense & 30 & 0 & 314995 & $\mathrm{O}$ \\
\hline PKMR115 & SRD5A3 & Chr4:56212560G $>$ A & NM_024592.4 & $c .57 \mathrm{G}>\mathrm{A}$ & p.Trp19* & Nonsense & NA & 357.1 & 611715 & c \\
\hline PKMR119 & PGAP1 & Chr2:197710616T $>C$ & NM_024989.3 & $c .2276 A>G$ & p.(Tyr759Cys) & Missense & 23.6 & 16.57 & 611655 & C \\
\hline PKMR133 & DOCK8 & Chr9:286599G $>A$ & NM_203447.3 & c.295G $>A$ & p.(Glu99Lys) & Missense & 25.9 & 247.3 & 611432 & c \\
\hline PKMR151 & TMEM67 & Chr8:94768056G > A & NM_153704.5 & c. $274 G>A$ & p.(Gly92Arg) & Missense & 25.6 & 17.41 & 609884 & $\mathrm{O}$ \\
\hline PKMR152 & WDR62 & Chr19:36587982G $>\mathrm{T}$ & NM_001083961.1 & c. $2520+1 G>T$ & p.(Leu840Leufs*95) & $\begin{array}{l}\text { Aberrant } \\
\text { splicing }\end{array}$ & NA & 0 & 613583 & C \\
\hline PKMR184 & SPG11 & Chr15:44876109delA & NM 025137.3 & c.5769delT & p.(Ser1923Argfs*28) & Frameshift & NA & 41.18 & 610844 & c \\
\hline PKMR188 & ASPA & Chr17:3402260G >A & NM_000049.2 & c. $820 \mathrm{G}>\mathrm{A}$ & p.(Gly274Arg) & Missense & 28.9 & 8.421 & 608034 & C \\
\hline PKMR193 & $A R L 13 B$ & Chr3:93755508G $>A$ & NM_182896.2 & C.599G $>\mathrm{A}$ & p.(Arg200His) & Missense & 34 & 8.314 & 608922 & c \\
\hline PKMR212 & ZFYVE26 & Chr14:68268804_05delGA & NM_015346.3 & c.1630_1631delTC & p.(Ser544Leufs*24) & Frameshift & NA & 8.236 & 612012 & C \\
\hline PKMR216 & $A P 4 S 1$ & Chr14:31539047A $>G$ & NM_007077.4 & c. $139-2 A>G$ & p. (GIn46GInfs*85) & $\begin{array}{l}\text { Aberrant } \\
\text { splicing }\end{array}$ & NA & 24.71 & 607243 & C \\
\hline PKMR224 & MKKS & Chr20:10393388delT & NM 018848.3 & c.775delA & p.(Thr259Leufs*21) & Frameshift & NA & 74.18 & 604896 & c \\
\hline PKMR242 & WDR73 & Chr15:85186864A $>G$ & NM_032856.3 & C.974T $>C$ & p.(Phe325Ser) & Missense & 29.9 & 0 & 616144 & $\mathrm{O}$ \\
\hline PKMR264 & $F R Y$ & Chr13:32747633G $>A$ & NM_023037.2 & $c .2281 \mathrm{G}>\mathrm{A}$ & p.(Val761lle) & Missense & 21.7 & 8.281 & 614818 & $\mathrm{O}$ \\
\hline PKMR281 & GPT2 & Chr16:46956326C $>T$ & NM_133443.3 & c. $1210 \mathrm{C}>\mathrm{T}$ & p. $($ Arg 404*) & Nonsense & NA & 33.31 & 138210 & $\mathrm{O}$ \\
\hline PKMR321 & FLNA & ChrX:153583356G $>A$ & NM_001110556.1 & $c .5054 \mathrm{C}>\mathrm{T}$ & p.Thr 1685 Met & Missense & 27.1 & 11.58 & 300017 & C \\
\hline
\end{tabular}

Abbreviations: C, concordant; D, discordant; NA, not applicable; O, overlapping features. ${ }^{a}$ Chromosomal position according to GRCh37/hg19. ${ }^{b}$ Novel mutations are written in bold font. ${ }^{\mathrm{C} C o n c o r d a n c e ~ o f ~ t h e ~}$ observed phenotype with the phenotype reported in Online Mendelian Inheritance in Man (OMIM). ${ }^{\mathrm{d}}$ Reported phenotypes show a dominant inheritance pattern, whereas the mutations reported here are homozygous. 


\begin{tabular}{|c|c|c|c|c|c|c|c|c|c|c|}
\hline Family & Ethnicity & Genes & Position $^{\mathrm{a}}$ & Transcript & cDNA mutation & Protein change & Variation & Protein function & $\begin{array}{c}\text { CADD } \\
\text { score v1.3 }\end{array}$ & $\begin{array}{c}\text { ExAC allele } \\
\text { frequency } \times 10^{-6}\end{array}$ \\
\hline PKMR24 & Punjabi & ZSCAN25 (ZNF498) & Chr7:99219114A>G & NM_145115.2 & c. $.506 \mathrm{~A}>\mathrm{G}$ & p.(Glu169Gly) & Missense & $\begin{array}{l}\text { Zinc finger protein nucleic } \\
\text { acid binding }\end{array}$ & 26.5 & 0 \\
\hline PKMR33 & Punjabi & DPH1 & Chr17:1944817C > T & NM_001383.3 & c. $1144 C>T$ & p.(Pro382Ser) & Missense & Diphthamide biosynthesis & 32 & 8.515 \\
\hline PKMR40 & Pathan & DCTN2 & Chr12:57929561T > G & NM_001261412.1 & c. $173 \mathrm{~A}>\mathrm{C}$ & p.(Lys58Thr) & Missense & Structural protein & 26.8 & 8.484 \\
\hline PKMR43 & Pathan & METTL5 & Chr2:170677663_64delTC & NM_014168.3 & c.344_345delGA & p.(Arg115Asnfs*19) & Frameshift & Methyl transferase & NA & 0 \\
\hline PKMR45 & Pathan & TANGO2 (C22orf25) & Chr22:20041047G > T & NM_152906.5 & c. $353 \mathrm{G}>\mathrm{T}$ & p.(Gly118Val) & Missense & Transport and Golgi organization & 29.4 & 0 \\
\hline PKMR64 & Punjabi & CAPN12 ${ }^{\mathrm{b}}$ & Chr19:39230761_62delTT & NM_144691.4 & c.658_659delAA & p.(Asn220Glnfs*25) & Frameshift & Cystine protease & NA & 795.1 \\
\hline PKMR66 & Punjabi & TBCID8 & Chr2:101652537G $>A$ & NM_001102426.1 & c. $1501 \mathrm{C}>\mathrm{T}$ & p.(Leu501Phe) & Missense & G-protein modulator & 31.0 & 99.37 \\
\hline PKMR67 & Punjabi & MSS51 (ZMYND17) & Chr10:75187870G >A & NM_001024593.1 & c. $173 \mathrm{C}>\mathrm{T}$ & p.(Ser58Leu) & Missense & Zinc finger transcription factor & 33.0 & 140.1 \\
\hline PKMR72 & Punjabi & $M D G A 2$ & Chr14:47343402T $>$ C & NM_001113498.2 & c. $2232 \mathrm{~A}>\mathrm{G}$ & p.(Arg744Arg) & Aberrant Splicing & GPI anchor & NA & 52.83 \\
\hline PKMR98 & Punjabi & $F M O D$ & Chr1:203316893C>T & NM_002023.4 & c.506G $>\mathrm{A}$ & p.(Arg169Gln) & Missense & Receptor & 23.3 & 296.6 \\
\hline PKMR118 & Punjabi & C22orf31 & Chr22:29454885C > G & NM_015370.1 & c.718G $>C$ & p.(Gly240Arg) & Missense & Unknown & 28.6 & 0 \\
\hline PKMR142 & Punjabi & SMARCA1 & ChrX:128657269C $>$ T & NM_139035.2 & c. $79 \mathrm{G}>\mathrm{A}$ & p.(Glu27Lys) & Missense & Component of NURF complex & 22.1 & 35.55 \\
\hline PKMR153 & Punjabi & GPAA1 & Chr8:145138854G >C & NM_003801.3 & c.527G $>C$ & p.(Trp176Ser) & Missense & Glycosylphosphatidylinositol & 27.6 & 16.58 \\
\hline PKMR155 & Kashmiri & OR2A12 & Chr7:143792562G $>A$ & NM_001004135.1 & c. $362 \mathrm{G}>\mathrm{A}$ & p.(Arg121Gln) & Missense & Olfactory receptor & 24.1 & 16.57 \\
\hline PKMR159 & Punjabi & AACS & Chr12:125612785A > G & NM_023928.3 & c.1388A > G; & p.(Asn463Ser); & Missense & Acetoacetyl-Co synthetase & 21.2 & 82.95 \\
\hline & & & Chr12:125621351C $>\mathrm{T}$ & & c. $1822 \mathrm{C}>\mathrm{T}$ & p.(Arg608Cys) & Missense & & 34.0 & 198.1 \\
\hline PKMR164 & Punjabi & GGN & Chr19:38877823G > A & NM_152657.3 & c. $79 \mathrm{C}>\mathrm{T}$ & p.(Arg27Cys) & Missense & Germ cell specific gene & 25.9 & 362.3 \\
\hline PKMR174 & Siraki & MEGF9 & Chr9: 123421769 C $>$ T & NM_001080497.2 & c. $686 \mathrm{G}>\mathrm{A}$ & p.(Gly229Asp) & Missense & Receptor & 26.8 & 59.54 \\
\hline PKMR195 & Siraki & WFDC1 & Chr16:84360517G $>A$ & NM_021197.3 & $c .634 \mathrm{G}>\mathrm{A}$ & p.(Gly212Arg) & Missense & Protease inhibitor & 24.2 & 65.89 \\
\hline PKMR198 & Punjabi & METTL4 & Chr18:2554909G $>\mathrm{T}$ & NM_022840.4 & c. $588 \mathrm{C}>\mathrm{A}$ & p.(Cys196*) & Nonsense & Methyl transferase & NA & 41.19 \\
\hline PKMR200 & Siraiki & $U B E 2 J 2$ & Chr1:1203295_96delTT & NM_194315.1 & c.77_78delAA & p.(Lys26Argfs*30) & Frameshift & Ubiquitin protein ligase & NA & 0 \\
\hline PKMR206 & Siraiki & CCDC82 & Chr11:96117539delC & NM_024725.3 & c.373delG & p.(Asp125llefs*6) & Frameshift & Unknown & NA & 0 \\
\hline PKMR213 & Siraiki Baloch & TMEM222 & Chr1:27657230G $>A$ & NM_032125.2 & c. $214 \mathrm{G}>\mathrm{A}$ & $\begin{array}{l}\text { p.(Gly72Ser) } \\
\text { (Glo }\end{array}$ & Missense & Transmembrane protein & 34.0 & 8.237 \\
\hline PKMR215 & Siraiki & PUS7 & chr7:105148870_71delTG & NM_019042.3 & c.89_90delCA & p.(Thr30Lysfs*20) & Frameshift & Pseudouridylate synthetase & NA & 8.237 \\
\hline PKMR258 & Punjabi & AREL1 1 & Chr14:75150203G $>$ A & NM_001039479.1 & c. $27 \overline{7} \mathrm{C}>\mathrm{T}$ & p.(His93Tyr) & Missense & Ubiquitin-protein ligase & 23.1 & 1102 \\
\hline PKMR298 & Punjabi & SEPT6 & ChrX:118797529T >C & NM_145799.3 & c. $257 \mathrm{~A}>\mathrm{G}$ & p.(Tyr86Cys) & Missense & Filament formation & 26.4 & 11.41 \\
\hline PKMR318 & Punjabi & DUOX1 & Chr15:45444197G $>\mathrm{T}$ & NM_ 175940.2 & c.3140G $>\mathrm{T}$ & p.(Cys 1047Phe) & Missense & Oxidase & 22.9 & 16.59 \\
\hline PKMR320 & Punjabi & SLC7A10 & Chr19:33700282_83dupA & NM_019849.2 & c.1372_1373dupA & p.(Thr458Asnfs*71) & Frameshift & Transporter & NA & 16.49 \\
\hline PKMR325 & Punjabi & $T M 2 D 3$ & Chr15:102182749G $>A$ & NM_078474.2 & $c .677 \mathrm{C}>\mathrm{T}$ & p.(Thr226Met) & Missense & Cell death or proliferation & 31.0 & 8.344 \\
\hline PKMR326 & Pathan & PRKAR2B & Chr7:106797706G > T & NM_002736.2 & c. $1060 \mathrm{G}>\mathrm{T}$ & p.(Ala354Ser) & Missense & Kinase modulator & 32.0 & 8.247 \\
\hline PKMR396 & Punjabi & $R G R$ & Chr10:86008779G $>A$ & NM_002921.3 & c. $350 \mathrm{G}>\mathrm{A}$ & p.(Arg117His) & Missense & $\begin{array}{l}\text { Retinal G-protein coupled } \\
\text { receptor }\end{array}$ & 26.5 & 107.3 \\
\hline
\end{tabular}


(5) pathogenicity prediction by multiple in silico programs; and (6) enrichment of loss-of-function (LOF) and potentially pathogenic variants of known and novel ID genes in patients with ID as compared with individuals with the non-ID phenotype $(n=213$; Supplementary Table S3).

Pathogenic variants identified in previously reported ARID genes We identified 34 predicted pathogenic variants in 32 genes previously associated with ID or related neurodevelopmental disorders (Tables 1 and 3a; Supplementary Figures S3 and S6). These variants include homozygous missense $(n=21)$, nonsense $(n=5)$, frameshift $(n=5)$ and splice site variants $(n=3)$. Twenty-five of these variants are novel (Tables 1 and 3a). All new variants segregated with the disease and were either absent in dbSNP142 or had an allele frequency $\leqslant 0.002$ in the ExAC Browser ${ }^{27}$ (Tables 1 and $3 a$ ).

Most genes were found to be mutated in only one family, suggesting a low incidence of founder mutations underlying ID in the Pakistani population. Two genes, TMEM67 and FRAS1, which carried different variants in two independent families (Tables 1 and 3a). Two families (PKMR61b and PKMR69) carry one homozygous variant each in two unrelated but known ID genes. PKMR61b has variants in TMEM67 and FGFR1 while PKMR69 has variants in FRAS1 and EXOSC8 (Table 3a; Supplementary Figure S6). No carriers of these variants, which were both predicted to be damaging and disease causing, were detected in our populationspecific controls. Two families each harbored a variant in an X-linked gene (ZNF41 and FLNA).

A comparison of the phenotypes of 34 variants in genes known to cause ID shows that in most families the phenotype is similar to the reported entity (Supplementary Table S4). Some deviations do occur, which may result from variable effects of allelic mutations or the effects of genetic modifier variants, which may be particularly relevant in populations with a high consanguinity rate. $^{28,29}$

Pathogenic variants identified in novel candidate ARID genes In 30 families we identified plausible pathogenic variants affecting a single gene that had not been previously implicated in ARID (Table 2; Supplementary Figure S4). The majority of these changes were missense variants $(n=23)$. In addition, we identified frameshift $(n=6)$, nonsense $(n=1)$ and splice site $(n=1)$ variants that result in a truncated protein or are predicted to result in nonsensemediated RNA decay. None of the variants were found in 213 unrelated, ethnically matched control individuals, indicating that they are not indigenous Pakistani polymorphisms. Clustal W (http://www.ebi.ac.uk/Tools/msa/clustalw2/) alignment of protein sequences encompassing the missense variants showed that most of the mutated amino acids are evolutionarily conserved (Supplementary Figure S5). In 9 of the families there were no notable clinical characteristics besides ID, whereas 21 families exhibited syndromic forms of ID (Supplementary Table S5).

In family PKMR159 a compound heterozygous change was detected in $A A C S$ and both variants have a frequency $<0.0002$ in the ExAC database ${ }^{27}$ (Table 2). In silico programs support a pathogenic nature of these variants, suggesting that both alleles are pathogenic and may contribute to the ID phenotype. In family PKMR72 a synonymous change (c.2232A $>$ G, p.(Arg813Arg)) was identified in MDGA2 in the acceptor splice site of exon 13, which is predicted to affect splicing by three splice prediction programs (NNSPLICE ${ }^{30}$, HSF $^{31}$ and MaxEntScan ${ }^{32}$ ).

In eight families, variants in more than one gene were identified (Tables 3a and 3b, ; Supplementary Figure S6). Four of these genes have already been implicated in a neurological disease (TMEM67, FGFR1, FRAS1 and EXOSC8), but most variants affect genes that have not previously been connected to human disease phenotypes. Of the 19 co-segregating variants, 18 are missense variants 
and 1 is a splice site mutation. The identification of multiple variants co-segregating with the disorder suggests the possible existence of composite phenotypes (Supplementary Table S6), which might be a relatively common phenomenon in consanguineous populations.

Next, to determine the functional effect of variants, especially missense, on the secondary structure, stability and interactions of novel candidate ID proteins, we performed in silico molecular modeling using the HOPE and Pyre2 web-based programs. On the basis of the amino-acid sequence and structural homology with proteins with resolved crystal structures, we evaluated the effect of novel missense alleles in new ID proteins (Supplementary Figure S7). The identified variants are predicted to alter the function of the encoded proteins through their effect (loss off ionic interactions, loss of external interactions, de-stabilization of the core or the provision of more rigidity) on the secondary structure (Supplementary Figure S7).

Newly identified ARID proteins participate in essential regulatory networks

Next, we applied Ingenuity Pathway Analysis ${ }^{33}$ to visualize the interactions between the 485 previously reported ID proteins (http://gfuncpathdb.ucdenver.edu/iddrc/iddrc/GeneQuest.php) and our novel candidate ID genes to identify the biological pathways underlying the disease process. The overall analysis, which includes physical interactions, co-expression, activation, inhibition and protein-RNA interactions, suggests that most novel ARID candidate proteins are part of already-established molecular ID gene networks (Figure 1b). By taking only physical interactions into account, we found the integration of several new ARID candidate proteins in oxidative phosphorylation, mitochondrial dysfunction, PTEN signaling and PPAR/RXR activation $(P<0.0001$; Figure 1a).

Functional co-expression networks of ARID genes

To gain insight into the spatio-temporal expression pattern of the new candidate ARID genes, we extracted the mRNAseq data from the Allen Brain Atlas ${ }^{34}$ and analyzed the expression patterns in developing and adult human brain tissues (Supplementary Tables S7 and S8). All new candidate ARID genes are expressed in the human brain from a very early development stage to adulthood (Supplementary Table S8).

When examining the co-expression functional networks for our genes from the BrainScan database, we find that they are significantly (Bonferroni-corrected $a \leqslant 0.0042$ ) co-expressed in the adult brain in all four brain regions and that they are significantly (Bonferroni-corrected $P$-value $<0.0001$ ) co-expressed in the temporal-parietal and sub-cortical regions from infancy to late childhood (Figure 2a). The phenotype observed in most of our ID families is established at birth, so to further evaluate coexpression during embryonic stages, we re-examined the normalized mRNAseq data at various post-conception weeks (p.c.w.). We found significant co-expression of ID genes in the frontal cortex region from 12 p.c.w. onwards (Figure 2b). Later in embryonic development (24-37 p.c.w.) ID genes are also co-expressed in the temporal-parietal and sub-cortical regions (Figure 2b), which persisted from infancy to late childhood (Figure 2a).

\section{DISCUSSION}

We report the results of ES in a cohort of 121 consanguineous Pakistani families. Likely causative variants in known ID genes were identified in 30 of these families. In addition, a single likely pathogenic variant affecting genes not previously implicated in ID was identified in 30 families, and 8 families had multiple variants segregating with the ID phenotype (Figure 3). Replication of this study in independent families is warranted to add these genes to 


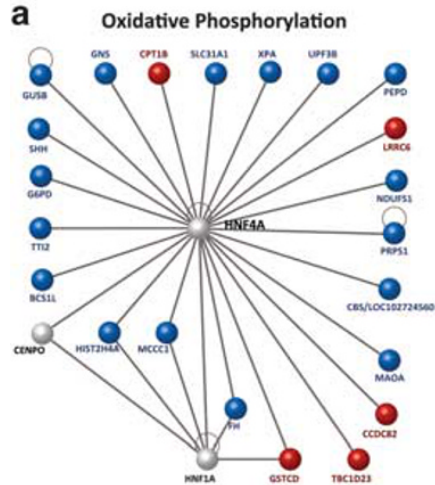

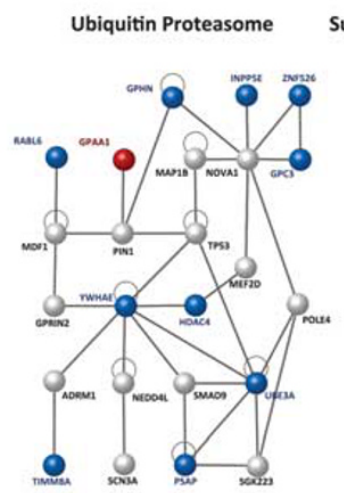

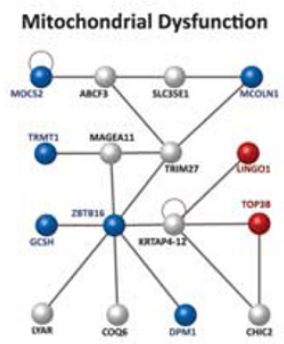

Superpathway of Citrulline Metabolism
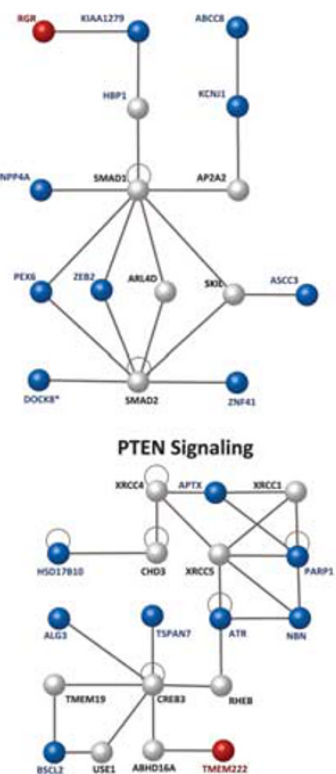

b

Molecular Network of new ID genes

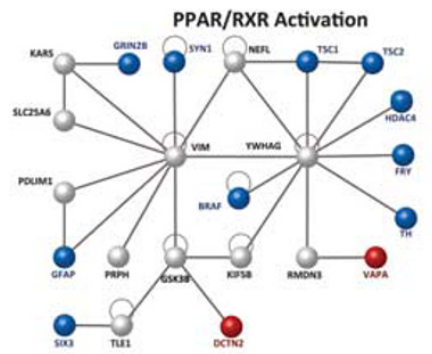

Figure 1. (a) Protein-protein interactions of novel candidates in different pathways. Novel candidate genes either directly interact with known intellectual disability (ID) genes (in blue) or through other genes (in gray) in the pathway. (b) Molecular functions of novel candidate genes (in red) for ID are indicated. Genes are involved in various cellular processes by activating, inhibiting and interacting with other molecules and are part of protein complexes.

the rapidly growing list of bona fide genes implicated in ID. In fact, during the submission phase of this manuscript-independent mutations in ARID phenotypes were already reported for two genes: DPH1 (ref. 35) and TANGO2. ${ }^{36,37}$ Other arguments that strengthen the involvement of the candidate ID genes are the disruptive nature of missense mutations as observed by modeling of the protein structure (Supplementary Figure S7), the cooccurrence of ARID candidate genes in common regulatory pathways (Figure 1) and the co-expression of these genes in distinct regions of the human brain (Figure 2). Further credit for the involvement of the new candidate ID genes is provided by the pattern of mutations. The frequency of predicted LOF alleles (that is, nonsense, frameshift or splice site variants) in known ID genes is $13 / 34$ (38.24\%), whereas their occurrence for new candidate genes is $8 / 30(26.67 \%)$, and only $1 / 15(6.67 \%)$ for candidate genes in families with multiple segregating variants. These LOF variants in the new candidate genes, METTL4, METTL5, CAPN12, MDGA2, UBE2J2, CCDC82, PUS7, SLC7A10 and TBC1D23, have the highest confidence of causality. In addition, we analyzed the exomes of 213 unrelated Pakistani control individuals for variants affecting the known ID genes and the novel candidate ID genes that we have identified in our cohort. Importantly, homozygous and compound heterozygous variants that would have passed our stringent filtering criteria were identified in eight different genes. Five of these are known ID genes (APTX, ASPM, FLNA, POMT2 and SYNE1), and only three are new candidate ID genes, of which two were found in families with multiple segregating variants (ESYT3 and SLC45A4) and one affecting a gene (GGN) that harbored the single segregating variant in the respective family. The relative high occurrence of LOF alleles in families with single segregating variants, as well as the paucity of predicted pathogenic variants in these genes in a control cohort provides strong support for the involvement of the candidate ID genes listed in Table 2.

Recently, ES of 3222 apparently healthy British adults of Pakistani heritage revealed 1111 rare homozygous variants in 781 genes, including a frameshift allele (c.658_659delAA) in one of the novel candidate genes CAPN12. ${ }^{38}$ No other homozygous LOF allele was observed in any novel ID candidate gene. We found the same frameshift allele (Table 2) segregating with a moderate ID phenotype (Supplementary Table S5) in two affected individuals of family PKMR64 (Supplementary Figure S4). There are at least three possible reasons to explain these observations. First, the LOF variant we identified in CAPN12 is not causative for the moderate ID phenotype in family PKMR64, and CAPN12 should be discarded as candidate ID gene. Second, without comprehensive assessment of mental health it is hard to rule out that the British-Pakistani individual has a mild ID phenotype similar to the phenotype in family PKMR64. Indeed, there are indications to question the health status of all individuals of the British-Pakistani cohort. For example, the reported genetic variants include homozygous LOF alleles in AHI1, C12orf57 and POMGNT1 genes, which are known to underlie the severe neurological disorders, Joubert syndrome ${ }^{39}$, Temtamy syndrome ${ }^{40}$ and muscle-eye-brain disease and other muscular dystrophy-dystroglycanopathies, ${ }^{41}$ respectively. Variants identified in the British-Pakistani adults also include LOF alleles in GJB2, MYO3A and $\mathrm{COCH}$ genes, which are known to cause hearing loss in humans. ${ }^{42-44}$ Third, there could be a protective or modifier genetic variant present in the British adult, which could render the effect of c.658 659delAA allele of CAPN12. ${ }^{45,46}$

In the remaining 53 families, no high-confidence DNA variants was identified. This might be accounted for by several factors. First, it is possible that some causative variants have not passed our stringent filtering criteria. Second, genetic heterogeneity is likely to occur, even within some of the families we have studied. Such phenocopies would disrupt the segregation of DNA variants across all affected individuals in the studied pedigrees. Third, it is possible that some of the phenotypes with mild to moderate ID are caused by the digenic or oligogenic inheritance of rare variants or polymorphisms with low effect size. Finally, some mutations may have escaped detection by ES and may reside in non-coding regions or regions poorly covered by exonic enrichment. 
a
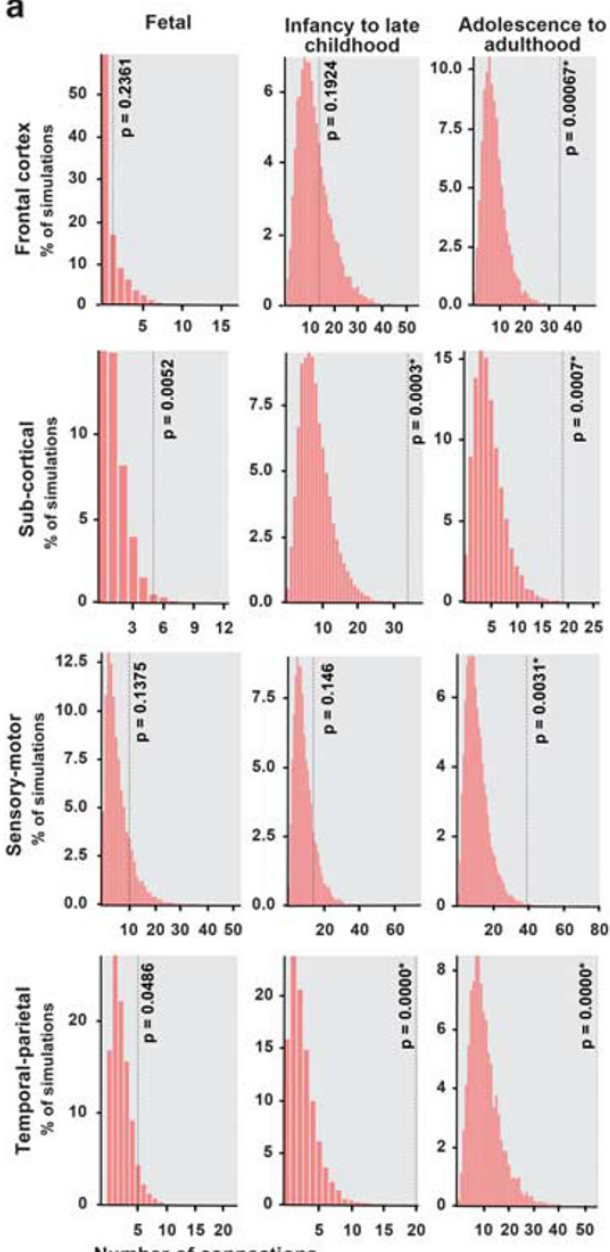

b
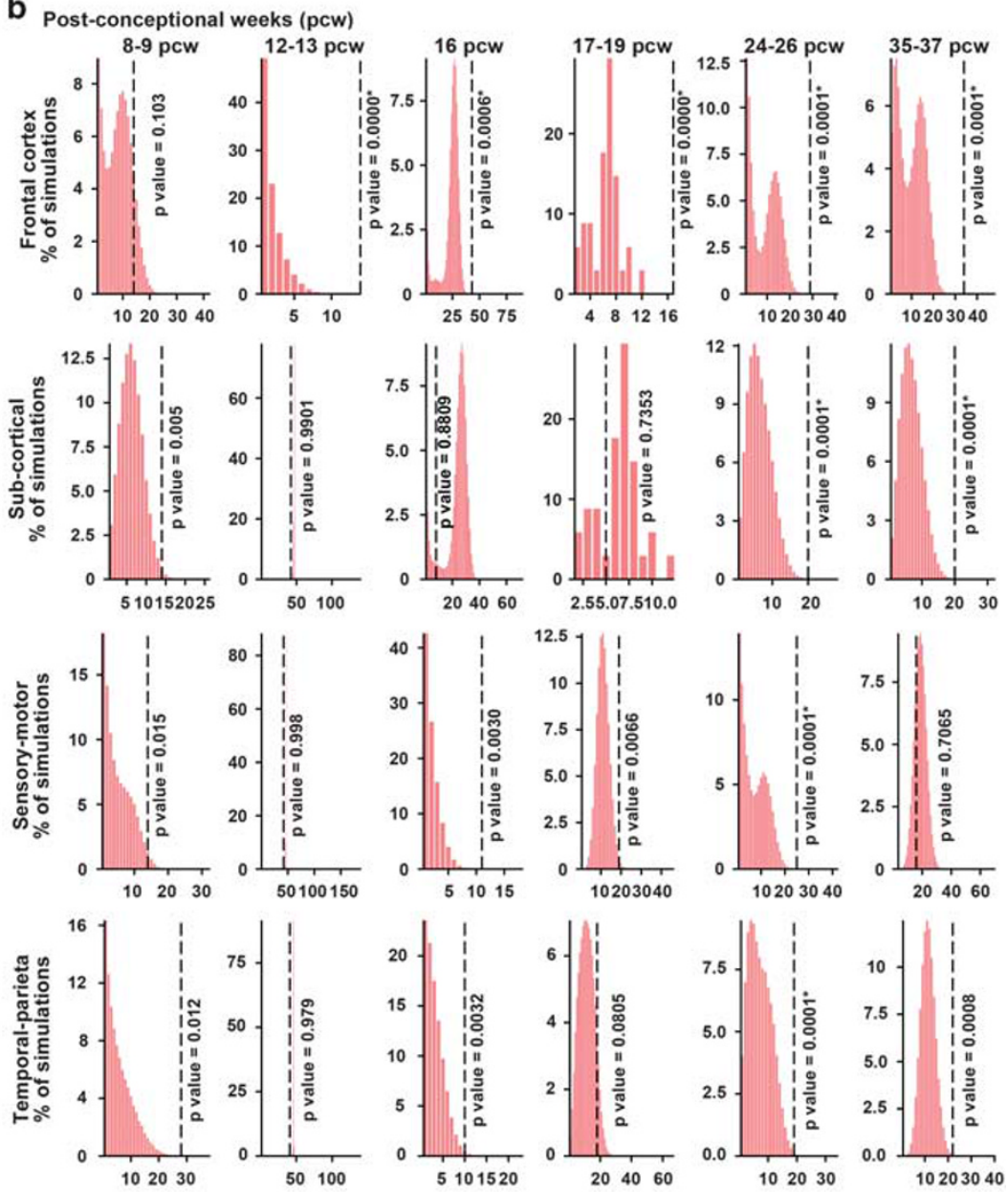

Figure 2. Co-expression of novel intellectual disability (ID) candidate genes was evaluated using RNA-seq data from the BrainSpan Atlas. (a) Gene pairs were defined as connected if the absolute value of the Pearson's correlation coefficient is $>0.8$ for their expression levels in different brain region (frontal cortex, temporal-parietal, sensory-motor and sub-cortical) and different developmental stage (fetal, infancy to late childhood and adolescence to adulthood). Connections of co-expression genes at three development stages and four brain regions were plotted. Dotted lines indicate numbers of connections (edges) in networks created using target genes. Histograms represent distributions of the numbers of edges in 10000 simulated networks using a similar number of random genes. ${ }^{*}$ Represents the significance enrichment with $P$ less than adjusted $P$-value. (b) Co-expression of novel ID candidate genes during embryonic developmental stages for the same four brain regions.

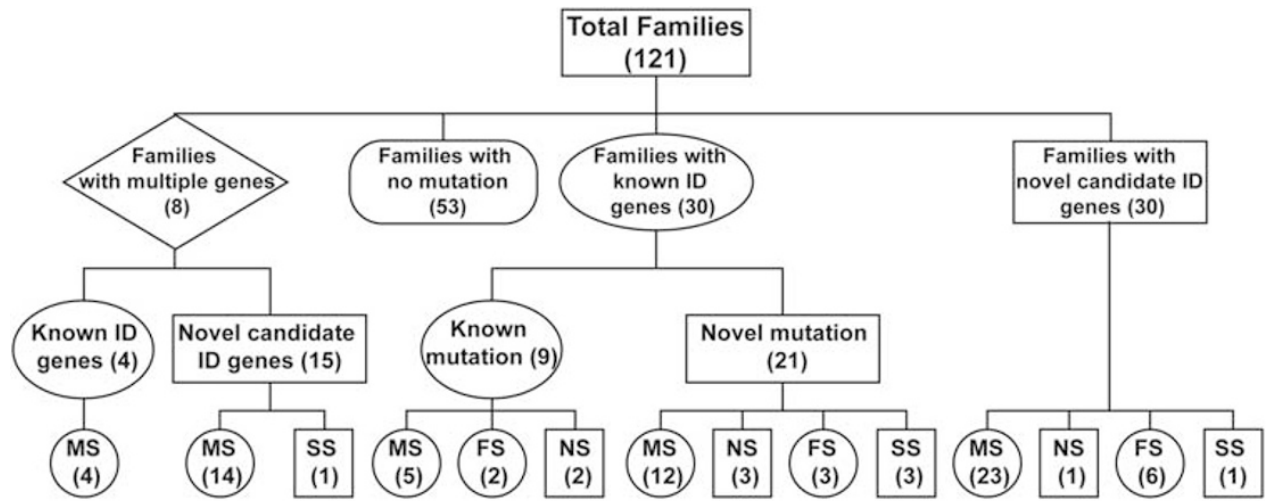

MS: missense; SS: splice site; FS: frameshift; NS: nonsense

Figure 3. Overview of the results of genetic studies in 121 consanguineous Pakistani families segregating recessive intellectual disability (ID) phenotype.

Intellectual disability and other neurodevelopmental disorders

There is a growing body of evidence that categorical neurodevelopmental disorders such as ID, autism spectrum disorders, attention deficit/hyperactivity disorders and learning disorders lack precise boundaries in their clinical definitions, epidemiology, genetics, and molecular and cellular networks. ${ }^{47-52}$ Accordingly, cognitive and 
behavioral comorbidities such as attention deficit/hyperactivity disorders, speech delay and aggression are also frequently seen in the ID families studied here. In our study, $21.88 \%$ of the families (7 of 32) with mutations in known genes (Supplementary Tables S4 and S6) and $33.33 \%$ of the families (12 of 36) with variants in novel candidate ARID genes resulting in clinical phenotypes that include epilepsy (Supplementary Tables S5 and S6). These results are not unexpected because the prevalence of epilepsy in individuals with some degree of ID ranges from 5.5 to $35 \% .{ }^{53}$ The prevalence of epilepsy grows with increasing severity of ID, with $15 \%$ of individuals with mild to moderate ID also exhibiting epilepsy and more than $30 \%$ of those with severe ID. ${ }^{54}$ This high prevalence underscores the importance of identifying largely unknown common genetic factors with a causative role in epilepsy and ARID. A striking example is provided by family PKMR82, in which a homozygous nonsense mutation (p.Arg65*) in KCNA2 was associated with mild to moderate ID, speech delay, strabismus, walking delay and epilepsy. Recently, de novo KCNA2 missense mutations were associated with epileptic encephalopathy, ataxia, variable $\mathrm{ID}^{55,56}$ and other features reminiscent of those found in family PKMR82. These de novo missense mutations exert dominant effects by gain-of-function and dominant-negative mechanisms. The nonsense mutation in PKMR82 represents the first true LOF allele of $K C N A 2$, which apparently has no phenotypic consequences in heterozygous mutation carriers.

\section{Combinatorial ID phenotypes}

We observed a syndromic ID phenotype in most of the studied families (Supplementary Tables S4-S6). These syndromic ID phenotypes could be attributed to the variant of a single gene in most families, but we have also observed multiple independently segregating variants in eight families (6.6\%) of our cohort (Tables $3 a$ and $3 b$ ). This high co-occurrence of multiple potential disease-causing alleles may lead to the occurrence of composite recessive phenotypes. Both families PKMR61b and PKMR69 have segregating variants in two independent known disease genes. On the basis of the clinical presentation of previous mutations, in families PKMR61b and PKMR69 it seems that the core phenotypic features are attributable to just one of the two variants: TMEM67 in family PKMR61b and EXOSC8 in family PKMR69. Such inferences cannot be made for families in which segregating variants are seen in multiple novel candidate ID genes. For example, in family PKMR131 independent variants in ESYT3 and CCT6B are associated with an unremarkable phenotype consisting of mild ID, speech delay, slow learning and aggressive behavior. CCT6B and ESYT3 have not been connected to any human disorder so far, and therefore the variants in either or both of these genes could contribute to the phenotype.

\section{Functional properties of novel ARID genes}

A comprehensive evaluation of the transcriptome profiles of normal human brain tissues revealed that the ID genes are significantly co-expressed in the frontal cortex region from 12 p.c.w. onwards, and in the temporal-parietal and sub-cortical regions from infancy to late childhood, implicating these regions in the pathogenesis of ID. These brain regions incorporate external and internal information and their disruptions have been associated with various disorders, such as amnesia, Alzheimer's disease and schizophrenia, ${ }^{57}$ which further support the notion of shared molecular networks between various brain disorders. At later stages, we also found significant co-expression in the frontal cortex and sensory-motor regions (Figure 2a), which suggests that many of the ID genes might also have an important role in postnatal mechanisms, such as fine-tuning of synaptic connectivity, circuitry formation, and acute synaptic and other neural processes. Evidence for the involvement of postnatal mechanisms in ID is provided by studies with conditional (postnatal) knockout mice, ${ }^{58,59}$ as well as studies with postnatal interventions in ID mouse models that are a basis of clinical trials for human ID disorders, such as Fragile $\mathrm{X}$ syndrome, Down syndrome and neurofibromatosis. ${ }^{60-62}$

\section{Common regulatory pathways involving new ARID genes}

Only few of the novel ID genes seem to relate to molecular pathways and networks that have been previously implicated in dominant and X-linked forms of ID, such as pre- and post-synaptic signaling, transcription regulation and epigenetic mechanisms. ${ }^{48,51,52}$ An exception is UBE2J2, encoding a ubiquitin-conjugating enzyme, which represents an emergent mechanism for regulating synapse function by post-translational modification through the ubiquitin pathway at the postsynaptic membrane. ${ }^{63}$ Proteolysis by the ubiquitin proteosome pathway is recognized as a major molecular pathway leading to several neurodevelopmental ${ }^{48}$ and neurodegenerative diseases. ${ }^{64}$ Previously identified gene networks that are commonly disrupted in ID and other neurodevelopmental disorders are composed of genes that are highly dosage-sensitive. Thus, these pathways might represent other neurobiological processes than those that are affected by recessive mutations.

\section{CONCLUSION}

Our study demonstrates the clinical utility of consanguineous populations for the elucidation of the molecular basis of very heterogeneous recessive disorders. Uncovering candidate genes for such disorders in inbred families will trigger the identification of matching mutations in other populations. In addition, a major strength of the present study is the presentation of the clinical profiles in conjunction with the reported candidate gene mutations. Therefore, the large collection of data presented in this manuscript is expected to facilitate the interpretation of DNA variants in diagnostic exome studies of patients with difficult-to-diagnose neurodevelopmental disorders. Future work using model systems may aid in unraveling and revealing the functional integration of different molecular networks in normal brain development and activity, which could add another level to neurological disorder diagnosis and more effective targeted therapy.

\section{CONFLICT OF INTEREST}

The authors declare no conflict of interest.

\section{ACKNOWLEDGMENTS}

We thank the patients and their family for participating in our study. Many thanks to $R$ Yousaf and S Yousaf (University of Maryland, MD, USA), WM Nillesen, K Neveling and Dr LE Vissers (Department of Human Genetics, Radboud University Medical Center, Nijmegen, The Netherlands) for technical and bioinformatics assistance. This study was supported by funds from the EU FP7 Large-Scale Integrating Project Genetic and Epigenetic Networks in Cognitive Dysfunction (241995 to HvB and SR), Higher Education Commission in Pakistan. Work in the US was supported by the funds from University of Maryland. DLP is recipient of a CAPES Fellowship (13311/131). This study made use of data generated by the UK10K Project. Funding for the UK10K Project was provided by the Wellcome Trust under award WT091310. A full list of consortium members can be found at the UK10K Project website (http://www. uk10k.org). We thank the Exome Aggregation Consortium and the groups that provided exome variant data for comparison. A full list of contributing groups can be found at http://exac.broadinstitute.org/about.

\section{AUTHOR CONTRIBUTIONS}

Conceived and designed the experiments: SR, ZMA, APMdB, HvB and ShR. Performed the experiments: MH, AR, ZI, MS, DLP, YS, EvB, LT-R, MR, MYZ, WMW-L, MA, ZA, KvH, FR, CG and APMdB. Analyzed the data: SR, MH, AR, ZI, MS, DLP, YS, AAK, KvH, WMW-L, MARB, MvdV, CG, MZA, DG, KC, FLR, TDO'C, SAR, SNK, ZMA, APMdB, HvB and ShR. Contributed reagents/materials/analysis tools: JAV, JA, TK, SAR, SNK, ZMA, APMdB, HvB and ShR. Wrote the paper: SR, APMdB, 
HvB and ShR. All the authors read the manuscripts and provided comments and have approved the final draft of the manuscript.

\section{REFERENCES}

1 Shaffer D, Campbell M, Bradley SJ, Cantwell DP, Carlson GA, Cohen DJ et al. Disorders usually first diagnosed in infancy, childhood, or adolescence. In: Ruth Ross, ed. Diagnostic and Statistical Manual of Mental Disorders: DSM-IV: International Version with ICD-10 Codes M.A. American Psychiatric Association: Washington, DC, 1995, pp 37-121.

2 Musante L, Ropers HH. Genetics of recessive cognitive disorders. Trends Genet 2014; 30: 32-39.

3 Ropers HH. Genetics of early onset cognitive impairment. Annu Rev Genomics Hum Genet 2010; 11: 161-187.

4 Maulik PK, Mascarenhas MN, Mathers CD, Dua T, Saxena S. Prevalence of intellectual disability: a meta-analysis of population-based studies. Res Dev Disabil 2011; 32: 419-436.

5 Leonard $\mathrm{H}$, Wen X. The epidemiology of mental retardation: challenges and opportunities in the new millennium. Ment Retard Dev Disabil Res Rev 2002; 8: 117-134.

6 Molinari F, Foulquier F, Tarpey PS, Morelle W, Boissel S, Teague J et al. Oligosaccharyltransferase-subunit mutations in nonsyndromic mental retardation. Am J Hum Genet 2008; 82: 1150-1157.

7 Gilissen C, Hehir-Kwa JY, Thung DT, van de Vorst M, van Bon BW, Willemsen MH et al. Genome sequencing identifies major causes of severe intellectual disability. Nature 2014; 511: 344-347.

8 Basel-Vanagaite L, Attia R, Yahav M, Ferland RJ, Anteki L, Walsh CA et al. The CC2D1A, a member of a new gene family with $\mathrm{C} 2$ domains, is involved in autosomal recessive non-syndromic mental retardation. J Med Genet 2006; 43: 203-210.

9 Higgins JJ, Pucilowska J, Lombardi RQ, Rooney JP. A mutation in a novel ATPdependent Lon protease gene in a kindred with mild mental retardation. Neurology 2004; 63: 1927-1931.

10 Molinari F, Rio M, Meskenaite V, Encha-Razavi F, Auge J, Bacq D et al. Truncating neurotrypsin mutation in autosomal recessive nonsyndromic mental retardation. Science 2002; 298: 1779-1781.

11 Najmabadi H, Hu H, Garshasbi M, Zemojtel T, Abedini SS, Chen W et al. Deep sequencing reveals 50 novel genes for recessive cognitive disorders. Nature 2011; 478: $57-63$

12 Alazami AM, Patel N, Shamseldin HE, Anazi S, Al-Dosari MS, Alzahrani $\mathrm{F}$ et al. Accelerating novel candidate gene discovery in neurogenetic disorders via wholeexome sequencing of prescreened multiplex consanguineous families. Cell Rep 2015; 10: 148-161.

13 Hosseini-Chavoshi M, Abbasi-Shavazi MJ, Bittles AH. Consanguineous marriage, reproductive behaviour and postnatal mortality in contemporary Iran. Hum Hered 2014; 77: 16-25.

14 Saadat M, Ansari-Lari M, Farhud DD. Consanguineous marriage in Iran. Ann Hum Biol 2004; 31: 263-269.

15 Bittles $\mathrm{AH}$, Black ML. The impact of consanguinity on neonatal and infant health. Early Hum Dev 2010; 86: 737-741.

16 lqbal Z, van Bokhoven $\mathrm{H}$. Identifying genes responsible for intellectual disability in consanguineous families. Hum Hered 2014; 77: 150-160.

17 Gustavson $\mathrm{KH}$. Prevalence and aetiology of congenital birth defects, infant mortality and mental retardation in Lahore, Pakistan: a prospective cohort study. Acta Paediatr 2005; 94: 769-774.

18 Zhang X, Snijders A, Segraves R, Zhang X, Niebuhr A, Albertson D et al. Highresolution mapping of genotype-phenotype relationships in cri du chat syndrome using array comparative genomic hybridization. Am J Hum Genet 2005; 76: 312-326.

19 Grimberg J, Nawoschik S, Belluscio L, McKee R, Turck A, Eisenberg A. A simple and efficient non-organic procedure for the isolation of genomic DNA from blood. Nucleic Acids Res 1989; 17: 8390.

20 Simon M, Richard EM, Wang X, Shahzad M, Huang VH, Qaiser TA et al. Mutations of human NARS2, encoding the mitochondrial asparaginyl-tRNA synthetase, cause nonsyndromic deafness and Leigh syndrome. PLoS Genet 2015; 11: e1005097.

21 Koressaar T, Remm M. Enhancements and modifications of primer design program Primer3. Bioinformatics 2007; 23: 1289-1291.

22 Szklarczyk D, Franceschini A, Wyder S, Forslund K, Heller D, Huerta-Cepas J et al. STRING v10: protein-protein interaction networks, integrated over the tree of life. Nucleic Acids Res 2015; 43: D447-D452.

23 Ulitsky I, Shamir R. Identification of functional modules using network topology and high-throughput data. BMC Syst Biol 2007; 1: 8 .

24 Ulitsky I, Maron-Katz A, Shavit S, Sagir D, Linhart C, Elkon R et al. Expander: from expression microarrays to networks and functions. Nat Protoc 2010; 5: 303-322.

25 Miller JA, Ding SL, Sunkin SM, Smith KA, Ng L, Szafer A et al. Transcriptional landscape of the prenatal human brain. Nature 2014; 508: 199-206.
26 Gulsuner S, Walsh T, Watts AC, Lee MK, Thornton AM, Casadei S et al. Spatial and temporal mapping of de novo mutations in schizophrenia to a fetal prefrontal cortical network. Cell 2013; 154: 518-529.

27 Exome Aggregation Consortium (ExAC) C, MA. Available at http://exac.broad institute.org (accessed on 15 January 2015).

28 Jordan DM, Frangakis SG, Golzio C, Cassa CA, Kurtzberg J, Task Force for Neonatal $\mathrm{G}$ et al. Identification of cis-suppression of human disease mutations by comparative genomics. Nature 2015; 524: 225-229.

$29 \mathrm{Vu} \mathrm{V}$, Verster AJ, Schertzberg M, Chuluunbaatar T, Spensley M, Pajkic D et al. Natural variation in gene expression modulates the severity of mutant phenotypes. Cell 2015; 162: 391-402.

30 Reese MG, Eeckman FH, Kulp D, Haussler D. Improved splice site detection in Genie. J Comput Biol 1997; 4: 311-323.

31 Desmet FO, Hamroun D, Lalande M, Collod-Beroud G, Claustres M, Beroud C. Human Splicing Finder: an online bioinformatics tool to predict splicing signals. Nucleic Acids Res 2009; 37: e67.

32 Yeo G, Burge CB. Maximum entropy modeling of short sequence motifs with applications to RNA splicing signals. J Comput Biol 2004; 11: 377-394.

33 Jimenez-Marin A, Collado-Romero M, Ramirez-Boo M, Arce C, Garrido JJ. Biological pathway analysis by ArrayUnlock and Ingenuity Pathway Analysis. BMC Proc 2009; 3(Suppl 4): S6.

34 Viggiano A, Cacciola G, Widmer DA, Viggiano D. Anxiety as a neurodevelopmental disorder in a neuronal subpopulation: evidence from gene expression data. Psychiatry Res 2015; 228: 729-740.

35 Loucks CM, Parboosingh JS, Shaheen R, Bernier FP, McLeod DR, Seidahmed MZ et al. Matching two independent cohorts validates $\mathrm{DPH} 1$ as a gene responsible for autosomal recessive intellectual disability with short stature, craniofacial, and ectodermal anomalies. Hum Mutat 2015; 36: 1015-1019.

36 Kremer LS, Distelmaier F, Alhaddad B, Hempel M, luso A, Kupper C et al. Bi-allelic Truncating Mutations in TANGO2 Cause Infancy-Onset Recurrent Metabolic Crises with Encephalocardiomyopathy. Am J Hum Genet 2016; 98: 358-362.

37 Lalani SR, Liu P, Rosenfeld JA, Watkin LB, Chiang T, Leduc MS et al. Recurrent muscle weakness with rhabdomyolysis, metabolic crises, and cardiac arrhythmia due to bi-allelic TANGO2 mutations. Am J Hum Genet 2016; 98: 347-357.

38 Narasimhan VM, Hunt KA, Mason D, Baker CL, Karczewski KJ, Barnes MR et al. Health and population effects of rare gene knockouts in adult humans with related parents. Science 2016; 352: 474-477.

39 Ferland RJ, Eyaid W, Collura RV, Tully LD, Hill RS, Al-Nouri D et al. Abnormal cerebellar development and axonal decussation due to mutations in AHI1 in Joubert syndrome. Nat Genet 2004; 36: 1008-1013.

40 Zahrani F, Aldahmesh MA, Alshammari MJ, Al-Hazzaa SA, Alkuraya FS. Mutations in c12orf57 cause a syndromic form of colobomatous microphthalmia. Am J Hum Genet 2013; 92: 387-391.

41 Yoshida A, Kobayashi K, Manya H, Taniguchi K, Kano H, Mizuno M et al. Muscular dystrophy and neuronal migration disorder caused by mutations in a glycosyltransferase, POMGnT1. Dev Cell 2001; 1: 717-724.

42 Kelsell DP, Dunlop J, Stevens HP, Lench NJ, Liang JN, Parry G et al. Connexin 26 mutations in hereditary non-syndromic sensorineural deafness. Nature 1997; 387: 80-83.

43 Robertson NG, Lu L, Heller S, Merchant SN, Eavey RD, McKenna M et al. Mutations in a novel cochlear gene cause DFNA9, a human nonsyndromic deafness with vestibular dysfunction. Nat Genet 1998; 20: 299-303.

44 Walsh T, Walsh V, Vreugde S, Hertzano R, Shahin $H$, Haika $S$ et al. From flies' eyes to our ears: mutations in a human class III myosin cause progressive nonsyndromic hearing loss DFNB30. Proc Natl Acad Sci USA 2002; 99: 7518-7523.

45 Chen R, Shi L, Hakenberg J, Naughton B, Sklar P, Zhang J et al. Analysis of 589,306 genomes identifies individuals resilient to severe Mendelian childhood diseases. Nat Biotechnol 2016; 34: 531-538.

46 Riazuddin S, Castelein CM, Ahmed ZM, Lalwani AK, Mastroianni MA, Naz S et al. Dominant modifier DFNM1 suppresses recessive deafness DFNB26. Nat Genet 2000; 26: 431-434.

47 Talkowski ME, Rosenfeld JA, Blumenthal I, Pillalamarri V, Chiang C, Heilbut A et al. Sequencing chromosomal abnormalities reveals neurodevelopmental loci that confer risk across diagnostic boundaries. Cell 2012; 149: 525-537.

48 van Bokhoven H. Genetic and epigenetic networks in intellectual disabilities. Annu Rev Genet 2011; 45: 81-104.

49 Adam D. Mental health: on the spectrum. Nature 2013; 496: 416-418.

50 Mullin AP, Gokhale A, Moreno-De-Luca A, Sanyal S, Waddington JL, Faundez V. Neurodevelopmental disorders: mechanisms and boundary definitions from genomes, interactomes and proteomes. Transl Psychiatry 2013; 3: e329.

51 McCarthy SE, Gillis J, Kramer M, Lihm J, Yoon S, Berstein Y et al. De novo mutations in schizophrenia implicate chromatin remodeling and support a genetic overlap with autism and intellectual disability. Mol Psychiatry 2014; 19: 652-658. 
52 De Rubeis S, He X, Goldberg AP, Poultney CS, Samocha K, Cicek AE et al. Synaptic, transcriptional and chromatin genes disrupted in autism. Nature 2014; 515: 209-215.

53 Berg AT, Plioplys S. Epilepsy and autism: is there a special relationship? Epilepsy Behav 2012; 23: 193-198.

54 Prince $\mathrm{E}$, Ring $\mathrm{H}$. Causes of learning disability and epilepsy: a review. Curr Opin Neurol 2011; 24: 154-158.

55 Syrbe S, Hedrich UB, Riesch E, Djemie T, Muller S, Moller RS et al. De novo loss- or gain-of-function mutations in KCNA2 cause epileptic encephalopathy. Nat Genet 2015; 47: 393-399.

56 Pena SD, Coimbra RL. Ataxia and myoclonic epilepsy due to a heterozygous new mutation in KCNA2: proposal for a new channelopathy. Clin Genet 2015; 87: e1-e3.

57 Abu-Akel A, Shamay-Tsoory S. Neuroanatomical and neurochemical bases of theory of mind. Neuropsychologia 2011; 49: 2971-2984.

58 Gemelli T, Berton O, Nelson ED, Perrotti LI, Jaenisch R, Monteggia LM. Postnatal loss of methyl-CpG binding protein 2 in the forebrain is sufficient to mediate behavioral aspects of Rett syndrome in mice. Biol Psychiatry 2006; 59: 468-476.

59 Schaefer A, Sampath SC, Intrator A, Min A, Gertler TS, Surmeier DJ et al. Control of cognition and adaptive behavior by the GLP/G9a epigenetic suppressor complex. Neuron 2009; 64: 678-691.

60 Berry-Kravis E. Mechanism-based treatments in neurodevelopmental disorders: fragile X syndrome. Pediatr Neurol 2014; 50: 297-302.
61 Gardiner KJ. Pharmacological approaches to improving cognitive function in Down syndrome: current status and considerations. Drug Des Devel Ther 2015; 9: 103-125.

62 van der Vaart T, Plasschaert E, Rietman AB, Renard M, Oostenbrink R, Vogels A et al. Simvastatin for cognitive deficits and behavioural problems in patients with neurofibromatosis type 1 (NF1-SIMCODA): a randomised, placebo-controlled trial. Lancet Neurol 2013; 12: 1076-1083.

63 Mabb AM, Ehlers MD. Ubiquitination in postsynaptic function and plasticity. Annu Rev Cell Dev Biol 2010; 26: 179-210.

64 Hegde AN, Upadhya SC. Role of ubiquitin-proteasome-mediated proteolysis in nervous system disease. Biochim Biophys Acta 2011; 1809: 128-140.

(c) (1) (2) This work is licensed under a Creative Commons AttributionNonCommercial-ShareAlike 4.0 International License. The images or other third party material in this article are included in the article's Creative Commons license, unless indicated otherwise in the credit line; if the material is not included under the Creative Commons license, users will need to obtain permission from the license holder to reproduce the material. To view a copy of this license, visit http:// creativecommons.org/licenses/by-nc-sa/4.0/

(c) The Author(s) 2017

Supplementary Information accompanies the paper on the Molecular Psychiatry website (http://www.nature.com/mp) 